\title{
Study on a New Type of Discrete Random Contention System based on Bina ry Tree Conflict Resolution Algorithm
}

\author{
Yingying Guo, Hong wei Ding, Yifan Zhao, Jing Nan, Donghuan Song, Ping ping Yu
}

\begin{abstract}
This paper proposed a new type of discrete random contention system based on the literature [1], researched it using the average cycle method, then get the formula of the systemic throughput for the new random access protocol by building mathe matical modeling; but the uncertainty of random multiple access on frequency and time resulting in the conflict probability increases as the higher loading, therefore, introducing binary tree conflict resolution algorithm is very important to improve the channel utilization. The paper analyzed the isolated binary tree conflict resolution algorithm and then got the average length of timeslot of the algorithm; on the basis, the paper puts forward a new type of discrete random contention system based on binary tree conflict resolution algorithm. Analytic formulate for throughput of the new discrete random contention system and the system based on the conflict resolution algorithm are derived by build ing mathematical model and using the average cycle method. Finally, the computer simulation experiments show that the correctness of the theoretical, at the same time, show that the new discrete random contention system protocol based on binary tree conflict resolution algorithm is effective to further improve the system performance.
\end{abstract}

Keywords: Kew type of discrete random contention system, isolated binary tree conflict resolution algorithm, throughput, system performance.

\section{Introduction}

In the process of rapid development of human society, the communication network also developed with it rapidly. Multiple access technology $[2,3]$ has been playing a key role in the rapid development of wireless communication network [5], and even a significant symbol of the updating of mobile communication. Whether it is in a wireless communication, satellite communication or in modern mobile communications, cluster communication, computer network communication system, when there are multiple data communication terminals communicate with other terminals through a common transmission channel simultaneously, it needs to select the appropriate multiple access technology according to their own situation. The multiple access communication [4] is usually divided into two

Y. Guo

School of Information, Yunnan University, Kunming, Yunnan, China

H. Ding $(\bowtie)$

Department of Communication Engineering, Yunnan University, Kunming, Yunnan, China

E-mail:dhw1964@163.com

Y. Zhao

School of Information, Yunnan University, Kunming, Yunnan, China

J. Nan

School of Information, Yunnan University, Kunming, Yunnan, China

D. Song

School of Information, Yunnan University, Kunming, Yunnan, China

P. Y

School of Information, Yunnan University, Kunming, Yunnan, China

Supported by the National Natural Science Foundation of Ch ina (61072079);

Natural Science Foundation of Yunnan Province (2010CD023);

Graduate Scientific Research Fund of Yunnan University (ynuy201047). 
categories: fixed allocation multiple access communication technology and random multiple access communication technology.

Random multiple access communication technology is an important wireless network communication technology protocol. In today's rapid development of a satellite communication system, packet rad io network and computer communications network, random contention multiple access control protocol get an increasing wide range of applications in the wireless communication network due to its effective utilization rate and s mall forwarding delay under a certain conditions. The advantage of random contention multiple access system is that the user can occupy public resources dynamically, thus, can make the most use of the existing data storage spaces and information transmission channels. However, because of the application of the share allocation strategy in multiple access system $[6,7,8,9]$, make the random multiple access system are not fixed in time and frequency range, result in some inevitable collisions in the transmission channels, and the conflicts are increase as the load aggravation. The conflicting information packets need to be retransmitted, this will cause the delay of the system increase, and the throughput is reduced. Therefore, using reasonable conflict resolution algorithm becomes a key issue to improve the performance of random contention mu ltiple access system.

In the various conflict resolution algorithms [11], the application of the binary tree conflict resolution [10, 12, 13, $14,15]$ has been remitted the occurrence of the collision to some extent, greatly improved the performance of the random multiple access system. Therefore, the paper is on the background of the direction of the laboratory investigation, takes the new discrete random contention system [16] with packet length is 2 as research objects, introduces the isolated binary tree conflict resolution algorithm, decomposing the impacted packets in the free timeslot, it imp roves the channel utilization and system throughput to s great extent.

\section{Analyses of Protocols}

\subsection{The new discrete random contention system}

The system model is shown as follows:

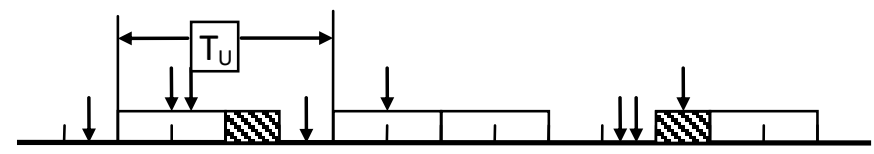

Fig. 1.1 Transmission mechanism of the new discrete random contention system

\subsubsection{Analysis of the new discrete system}

The assumptions are shown as follows before analyze the new system

1. The information packets arrival on the channel follows the Poisson distribution with an independent parameter $\mathrm{G}$, where $\mathrm{G}$ is arrival rate.

2. The channel is ideal and without any noise and interference.

3. Assume that the channel is divided into isometric TS on the time axis, and the len gth of each timeslot equals unit length.

4. Assume packets arrived during idle time or collision time collides on the channel, then the impacted packets stop sending information at end of the timeslot.

5. Packets arrived during the channel is busy to trans mit information are remitted to the beginning of next timeslot to transmit.

6. The length of successful packet is 2 , and the length of impacted packet or idle times lot is unit length. The variables are assumed in the following before making mathematical analysis

1. U represents an event that the information packet transmit successfully, B represents a pileup on the channel, I expresses the channel is in idle time, BI represents a joint event of B and I.

2. $p\left\{N_{B I}=i\right\}$ represents the probability of $\mathrm{i}$ joint events appear continuously on the channel in a cycle period . $p\left\{N_{U}=j\right\}$ represents the probability $\mathrm{j}$ successful events appear continuously on the channel in a cycle period. 
$p\left\{N_{U}=j, N_{B I}=i\right\}$ represents a joint probability appear i joint events and $\mathrm{j}$ successful events continuously in a cycle period.

3. S denotes throughput of a system. And

$$
S=\frac{E(U)}{E(B I)+E(U)}
$$

\subsubsection{Calculate the system throughput}

The transmission probabilities of information packet in the above random contention systems are shown in the following two:

1. Packets arrived during idle time or collision time.

The probability that the channel is idle in a timeslot is

$P_{A}(0)=e^{-G}$

The probability that information packets transmitted successfully in a timeslot is

$P_{A}(1)=G e^{-G}$

The probability that has x packets collided in a timeslot is

$P_{A}(x \geq 2)=\frac{G^{x} e^{-G}}{x !}$

2. Packets arrived during the channel is busy to transmit packets success fully.

The probability that the channel is idle in a times lot is

$P_{B}(0)=e^{-2 G}$

The probability that information packets transmitted successfully in a times lot is

$P_{B}(1)=2 G e^{-2 G}$

The probability that has x packets collided in a timeslot is

$P_{B}(x \geq 2)=\frac{(2 G)^{x} e^{-2 G}}{x !}$

The average number of timeslots that packets transmitted success fully in a cycle period is

$E\left(N_{u 2}\right)=\frac{2}{1-G e^{-G}}$

The average number of timeslots that packets collided or the channel is idle in a cycle period is

$E\left(N_{B I 2}\right)=\frac{1-2 G e^{-G}}{G e^{-G}\left(1-G e^{-G}\right)}$

Then average probability that packets transmitted success fully in a timeslot is

$P_{1}=\frac{G e^{-G}\left(1+2 G e^{-2 G}-2 G e^{-G}\right)}{1-G e^{-G}}$

The average probability that the channel is idle in a time slot is

$P_{0}=\frac{e^{-G}\left(1+2 G e^{-2 G}-2 G e^{-G}\right)}{1-G e^{-G}}$

The joint probability is

$P(i, j)=\left(\frac{e^{-G}\left(1+2 G e^{-2 G}-2 G e^{-G}\right)}{1-G e^{-G}}\right)^{i}\left(1-\frac{e^{-G}\left(1+2 G e^{-2 G}-2 G e^{-G}\right)}{1-G e^{-G}}\right)^{j}$

The average number of success ful event in a cycle period is

$$
E\left(N_{u}\right)=\frac{P_{1}}{P_{1}\left(1-P_{1}\right)}=\frac{1-G e^{-G}}{1-2 G e^{-G}\left(1-G e^{-G}+G e^{-2 G}\right)}
$$

The average number of collided or idle event in a cycle period is 


$$
E\left(N_{B I}\right)=\frac{1}{P_{1}}=\frac{1-G e^{-G}}{G e^{-G}\left(1-2 G e^{-G}+2 G e^{-2 G}\right)}
$$

The average length of success ful packets in a cycle period is

$$
E(U)=\frac{2\left(1-G e^{-G}\right)}{1-2 G e^{-G}\left(1-G e^{-G}+G e^{-2 G}\right)}
$$

The average length of collided or id le event in a cycle period is

$$
E(B I)=\frac{1-G e^{-G}}{G e^{-G}\left(1-2 G e^{-G}+2 G e^{-2 G}\right)}
$$

The throughput of system is

$$
S_{1}=\frac{2 G e^{-G}\left(1+2 G e^{-2 G}-2 G e^{-G}\right)}{1+2 G^{2} e^{-3 G}-2 G^{2} e^{-2 G}}
$$

\subsection{Analysis of Isolated Binary Tree Conflict Resolution Algorithm}

The binary tree is an all-important structure, and many data structure abstracted from practical problems is often the form of a binary tree, even a general tree can simply convert a binary tree structure, and its algorithm and storage structures are relatively simple, then the binary tree is especially important. The binary tree is a finite set of $n(n \geq 0)$ nodes, or an empty set $(n=0)$, or consists of a root node and two nodes, called left sub tree and right sub tree of the binary tree, which are disjo int.

\subsubsection{Conditions of the Algorithm}

(1) Packets transmitted on the channel are activated according to Poisson distribution, the activation rate is G, and the number of terminals, which need to send packets, are independent each other in any timeslot.

(2) The number of nodes in the system is not limited; all nodes sharing a common channel, the channel information are fed back to channel in the first time; each node send information in the beginning of a timeslot, and can confirm the channel status (idle, success, collision) in a fixed time t.

\subsubsection{Rules of the Algorithm}

(1) The in formation sources, which send message packets in the same timeslot, are grouped together.

(2) Each activated source belongs to the same group do Bernoulli experiments independently. If the result is 0 , select the left slot to transmit information, and if the result is 1 , then select the right.

(3) The information source occupied left slot separately sends information packets successfully, and stops doing Bernoulli experiments. Otherwise, repeat the process (1), (2), and turn to next step until all impacted packets retransmitted success fully in the left slot.

(4) The information source occupied right slot separately sends information packets successfully, and stops doing Bernoulli experiments. Otherwise repeat process (1) (3), until the nu mber of impacted packets is zeros.

Decomposition process of information packets for the algorith $\mathrm{m}$ is as shown in Fig. 1.2.

$$
\begin{aligned}
& P_{N 0}=2 ! C_{N}^{N} C_{0}^{0} P^{N} \\
& P_{N-1,1}=2 ! C_{N}^{N-1} C_{1}^{1} P^{N} \\
& P_{N 0}^{*}=1-P_{N 0}
\end{aligned}
$$

The average number of timeslots in demand is

$$
\bar{L}_{N}=\left(2+\bar{L}_{N-1}\right) P_{N-1,1}+\left(2+\bar{L}_{N}\right) P_{N 0}+\sum_{i=2}^{N / 2}\left(2+\bar{L}_{N-i}+\bar{L}_{i}\right) P_{N-i, i}
$$




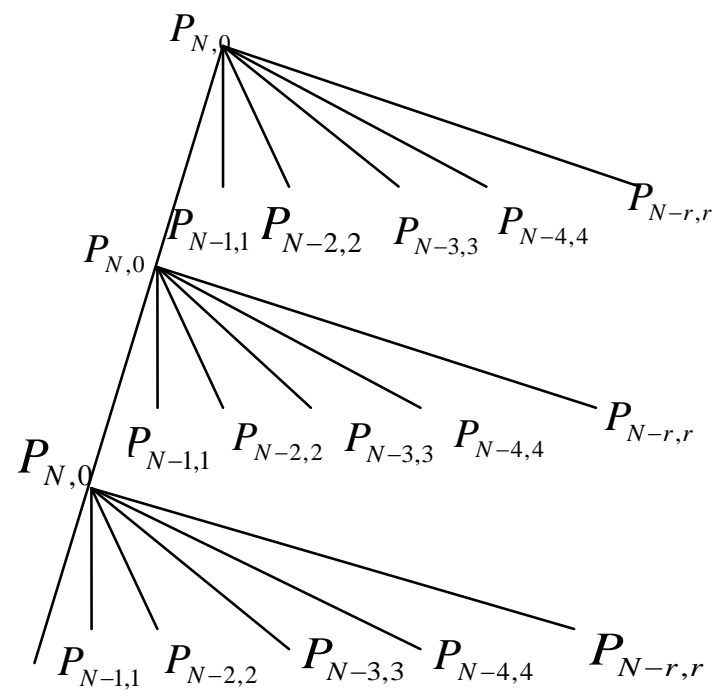

Fig. 1.2 The decomposition diagram of isolated binary tree conflict resolution algorithm

When $p=0.5$, the theoretical value of isolated binary conflict resolution algorithm in need is shown in table 1.1 , and computer simulation is also shown is the table.

Table 1.1 Theoretical value and experiment value of the average number of timeslots

\begin{tabular}{llllll}
\hline $\mathrm{N}$ & Theoretical value & Simulation value & $\mathrm{N}$ & Theoretical value & Simulation value \\
\hline 2 & 4.0000 & 3.9718 & 11 & 29.738 & 29.748 \\
3 & 6.6666 & 6.6638 & 12 & 32.624 & 32.606 \\
4 & 9.5238 & 9.4988 & 13 & 35.510 & 35.530 \\
5 & 12.419 & 12.424 & 14 & 38.395 & 38.398 \\
6 & 15.313 & 15.275 & 15 & 41.281 & 41.278 \\
7 & 18.201 & 18.177 & 16 & 44.167 & 44.068 \\
8 & 21.085 & 21.063 & 17 & 47.052 & 47.067 \\
9 & 23.969 & 23.938 & 18 & 49.937 & 49.944 \\
10 & 26.853 & 26.850 & 19 & 52.823 & 52.823 \\
\hline
\end{tabular}

As can be seen in the table, the simulation values and theoretical values of the average number of timeslots that is olate binary tree conflict resolution algorithm need to decompose vary considerably small, the increase of $n$, the closer of the simulations and theoretical values, and verify the correct of the theory.

\subsection{Performance Analysis of New Random Contention System based on Isolated Binary Tree Conflict Resolution Algorithm}

Build the as sociation random multip le access models combined binary conflict resolution algorithm and new random contention system with packet length 2 , describe and analyze it using average cycle method.

\subsubsection{Calculate the mean value}


The joint probability of $\mathrm{U}$ and $\mathrm{BI}$ event is

$P(i, j)=\left(\frac{e^{-G}\left(1+2 G e^{-2 G}-2 G e^{-G}\right)}{1-G e^{-G}}\right)^{i}\left(1-\frac{e^{-G}\left(1+2 G e^{-2 G}-2 G e^{-G}\right)}{1-G e^{-G}}\right)^{j}$

The number of idle slots is

$E\left(N_{I}\right)=\frac{P_{0}}{P_{1}\left(1-P_{1}\right)}=\frac{\left(1-G e^{-G}\right)}{G\left(1-2 G e^{-G}\left(1+G e^{-2 G}-G e^{-G}\right)\right)}$

Assume there are $x(x \geq 2)$ impacted packets in a timeslot, and then the number of timeslots that has $\mathrm{x}$ packets collide in a cycle period is

$E\left(N_{B x}\right)=\frac{P_{x}}{P_{1}\left(1-P_{1}\right)}=\frac{\left(1-G e^{-G}\right)\left(G^{x-1}\left(1-2 G e^{-G}\right)+(2 G)^{x} e^{-2 G}\right)}{x ! G e^{-G}\left(1-2 G e^{-G}+2 G e^{-2 G}\right)\left(1-2 G e^{-G}\left(1+G e^{-2 G}-G e^{-G}\right)\right)}$

The average length of idle slot in a cycle period on the channel is

$$
E(I)=\frac{\left(1-G e^{-G}\right)}{G\left(1-2 G e^{-G}\left(1+G e^{-2 G}-G e^{-G}\right)\right)}
$$

The average length of success ful packet is

$$
E(U)=\frac{2\left(1-G e^{-G}\right)}{1-2 G e^{-G}\left(1-G e^{-G}+G e^{-2 G}\right)}
$$

The average length of that has x packet impact on the channel is

$$
E\left(B_{x}\right)=E\left(N_{B x}\right) x
$$

$$
\begin{aligned}
& =\frac{\left(1-G e^{-G}\right)\left(G^{x-1}\left(1-2 G e^{-G}\right)+(2 G)^{x} e^{-2 G}\right)}{(x-1) ! G e^{-G}\left(1-2 G e^{-G}+2 G e^{-2 G}\right)\left(1-2 G e^{-G}\left(1+G e^{-2 G}-G e^{-G}\right)\right)} \\
& E\left(B_{x}^{*}\right)=E\left(N_{B x}\right)\left(1+\overline{L_{x}}\right) \\
& =\frac{\left(1-G e^{-G}\right)\left(G^{x-1}\left(1-2 G e^{-G}\right)+(2 G)^{x} e^{-2 G}\right)\left(1+\overline{L_{x}}\right)}{(x) ! G e^{-G}\left(1-2 G e^{-G}+2 G e^{-2 G}\right)\left(1-2 G e^{-G}\left(1+G e^{-2 G}-G e^{-G}\right)\right)}
\end{aligned}
$$

\subsubsection{Calculate the throughput}

According to the definition of system throughput, the throughput of the system with conflict resolution is

$$
S_{2}=\frac{E(U)+\sum_{x=2}^{\infty} E\left(B_{x}\right)}{E(I)+E(U)+\sum_{x=2}^{\infty} E\left(B_{x}^{*}\right)}
$$

Combines the formulas (2.1), (2.2), (2.3, (2.4) and (2.5), we conclude

$$
S_{2}=\frac{4 G e^{-G}-\left(4 G^{2}+2 G\right) e^{-2 G}+4 G^{2} e^{-3 G}+e^{G}-1}{2 e^{-G}-(2 G+1) e^{-2 G}+2 G e^{-3 G}+\frac{1-e^{-G}}{G}-1+\frac{1-2 G e^{-G}}{G} \sum_{x=2}^{\infty} \frac{G^{x} \overline{L_{x}}}{x !}+e^{-2 G} \sum_{x=2}^{\infty} \frac{(2 G)^{x} \overline{L_{x}}}{x !}}
$$

$\overline{L_{x}}$ is the desired average length of timeslot that the collisions being decomposed successfully by the isolated binary tree conflict resolution algorithm.

From literature [1], the throughput of traditional discrete rando m contention system is $S_{3}=G e^{-G}$ 


\section{Simulation Expe riment of the Control Protocol}

The simulation experiments of isolated binary tree conflict resolution algorith $\mathrm{m}$ based on the new random contention system is based on the above theoretical analysis and Matlab. Assume that the channel is in an idle state in the simulation process, that is there is no any interference and noise on the channel; the arrival rate of information packets is G; the length of timeslot is unit length, the length of successful packet is 2 and the length of the collision equals to the length of idle timeslot, is unit length.

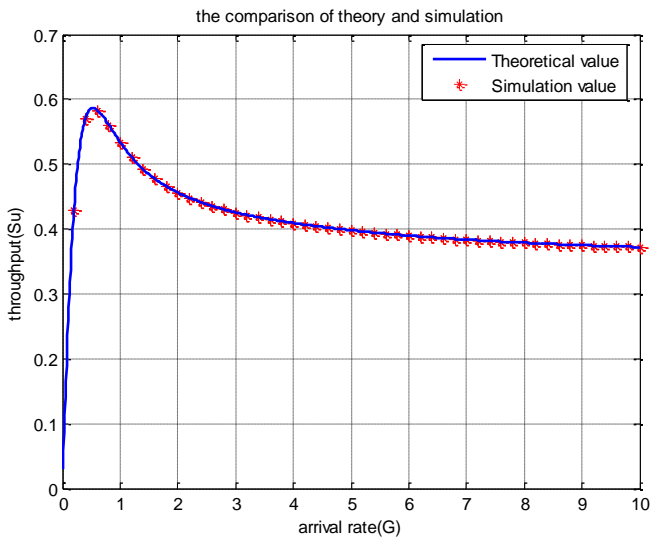

Fig. 1.3 the comparison of theory and simulation of the new random contention system based on isolated binary conflict resolution algorithm

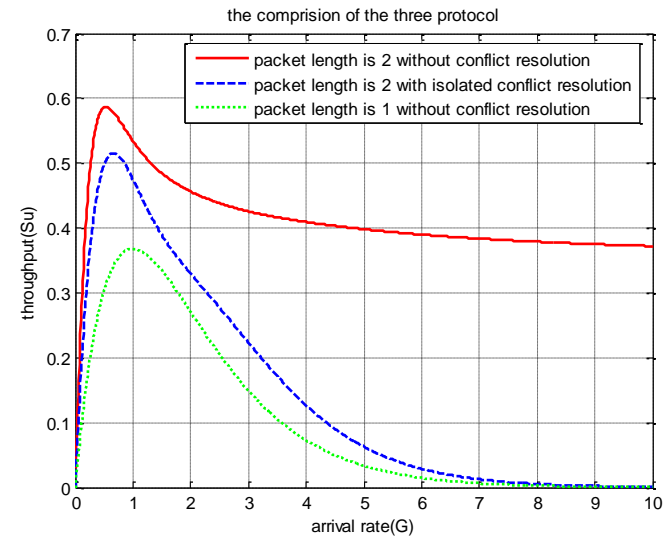

Fig. 1.4 the comparison of three protocols

(1) From Fig. 1.3 you can see, the theoretical value and simu lation value of throughput for the association random multiple access protocol combined binary tree conflict resolution algorithm and new random contention system coincide with each other to prove the correctness of the theory.

(2) You can see from Fig. 1.4, the new random contention access protocol with packet length is 2 greatly improve throughput than traditional slotted ALOHA, and the new random contention system based on binary tree conflict resolution algorith malso improve throughput than the new random contention protocol with packet length is 2 .

(3) Whether traditional slotted ALOHA protocol, or new discrete random contention system, the throughput approaches aero with heavy load, that is, the slotted ALOHA protocol is mainly in blocked state in the case of heavy load, the new random multiple access protocol based on binary tree conflict resolution algorithm, even under heavy load, the throughput has been maintained at about 0.368 .

\section{Conclusions}

This paper mainly introduced a new random contention system, the mechanism of isolated binary tree conflict resolution algorith $\mathrm{m}$ and a new discrete random access protocol based on binary tree conflict resolution algorith $\mathrm{m}$. By building mathematical model and using, mathematical methods to analyze and simulate the new random discrete random access protocol based on binary tree conflict resolution algorithm, and analy ze performance of the throughput that reflected on the two curves. We know that the performance of system has been greatly improved when introduce the isolated binary tree conflict resolution algorithm. However, due to invalid decomposition in the decomposition process of isolated binary tree conflict resolution algorithm makes the change of performance is still limited. Therefore, we can try to introduce the improved binary tree conflict resolution algorithm, or even $\mathrm{N}$-tree conflict resolution algorithm to random contention system and its performance is bound to get better improvement. The inadequacy of the system is to increase the complexity. 
Corres ponding Author Hongwei Ding (1964-), male, Professor of Yunnan University, PhD Degree. Mainly engaged in the research of random multiple access communication system, polling system, network communication engineering.

Acknowledgements This work was supported by the National Natural Science Foundation of Ch ina (61072079); Natural Science Foundation of Yunnan Province (2010CD023); Graduate Scientific Research Fund of Yunnan University (ynuy201047). Thanks for the seriously guidance of Professor Dongfeng Zhao.

\section{References}

[1] Zhao Dongfeng, Li Bihai, Zheng Sumin, Sun Quan. Study of Mean Cycle Times for Slotted ALOHA System. Journal of Yunnan University. 1994, 16(2): 133- 138.

[2] Zhao Dongfeng, Li Bihai, Zheng Sumin. Study on a New Method for the Slotted Access Channel. Journal of Electronics \& Information Technology, 1997, 19 (6): 814-819.

[3] Zhao Dongfeng. Study on the Average Cycle Method for Slotted Multiple Access Communications. Journal of China Institute of Communications, 1999, 20 (8): 80-85.

[4] Yu Ying, Zhao Dongfeng, Wang Dadong. Study on Two Average Cycle Schemes for Slotted Random Multi-access Channel. Journal of Yunnan Normal University Sciences Edition, 1998, 18(2): 20-23.

[5] Dong Geun Jeong. Performance of an exponential back off scheme for slotted-ALOHA protocol in local wireless environment. Vehicular Technology, IEEE Transactions on Industrial, 1995, 470- 479.

[6] Sallent, O.; Agusti, R.A proposal for an adaptive S-ALOHA access system for a mobile CDMA environment, Vehicular Technology, IEEE Transactions on Industrial, 1998.977- 986.

[7] Yingbo Hu, Weiwei Yang and Yueming Cai. Throughput analysis of slotted ALOHA with cooperative transmission using successive interference cancellation. Science in China Series F: Information Science.2009.2354-2359.

[8] Zhao Dongfeng, Zheng Sumin. Analysis of a Slotted Access Channel with Average Cycle Method, Proceedings of 1996 International Conference on Communication Technology. Beijing, China, Mary 5-7, 1996, 33.04.1- 33.04.4.

[9] A. El-Hoiydi. ALOHA with preamble sampling for sporadic traffic in ad hoc wireless sensor networks. IEEE International Conference on Communications (ICC), New York, Apr. 2002.

[10] Zhao Dongfeng, Li Bihai, Zheng Sumin. Study on Two-ray Tree Algorithms of Packet Conflict Resolution for Random Access Channels. Journal of University of Electronic Science and Technology of China. 1996, 25(2): 261-264.

[11] Gao Fei, Guo Yanping, Zhao Dongfeng. Analysis of Improved Contention Resolution Algorithms and Throughput over RAC. 2001, 30(2): 124-129.

[12] Hou Jianbin. Study on S-ALOHA based on Unfenced Two-ray Tree Algorithm of Packet Conflict Resolution. Yunnan University, 2011

[13] Xu Bo. Analysis on 1-persistent CSM A protocol based on Tree Algorithm of Packet Conflict Resolution. Yunnan University, 2011.

[14] Gao Yangsh ui. 1-insist CSMA and Tree Conflict Decomposition Algorithm Convergence Protocol and Application circuit Design. Yunnan University, 2012.

[15] Shivendra S P, ET all Collision resolution algorithms for a time2constrained multi2access channellIEEE Trans Commun, 1993, COM 241 (7): $1023-1027$.

[16] Yingtu W, Jinfu C1Collision resolution for variable2length messages1 IEEE Trans-Communication 1993, COM 241 (9): 1281 -1283. 